\title{
CRITICAL ANALYSIS OF BARYON MASSES AND SIGMA-TERMS IN HEAVY BARYON CHIRAL PERTURBATION THEORY *
}

\author{
Véronique Bernard \\ Centre de Recherches Nucléaires et Université Louis Pasteur de Strasbourg \\ Physique Théorique, Bat. 40A, BP 20, 67037 Strasbourg Cedex 2, France \\ Norbert Kaiser \\ Physik Department T30, Technische Universität München \\ James Franck Straße, W-8046 Garching, Germany \\ Ulf-G. Meißner ${ }^{\dagger}$ \\ Universität Bern, Institut für Theoretische Physik \\ Sidlerstr. 5, CH-3012 Bern, Switzerland
}

\begin{abstract}
We present an analysis of the octet baryon masses and the $\pi N$ and $K N \sigma$-terms in the framework of heavy baryon chiral perturbation theory. At next-to-leading order, $\mathcal{O}\left(q^{3}\right)$, knowledge of the baryon masses and $\sigma_{\pi N}(0)$ allows to determine the three corresponding finite low-energy constants and to predict the the two $K N \sigma$-terms $\sigma_{K N}^{(1,2)}(0)$. We also include the spin-3/2 decuplet in the effective theory. The presence of the nonvanishing energy scale due to the octet-decuplet splitting shifts the average octet baryon mass by an infinite amount and leads to infinite renormalizations of the low-energy constants. The first observable effect of the decuplet intermediate states to the baryon masses starts out at order $q^{4}$. We argue that it is not sufficient to retain only these but no other higher order terms to achieve a consistent description of the three-flavor scalar sector of baryon CHPT. In addition, we critically discuss an SU(2) result which allows to explain the large shift of $\sigma_{\pi N}\left(2 M_{\pi}^{2}\right)-\sigma_{\pi N}(0)$ via intermediate $\Delta(1232)$ states.
\end{abstract}

BUTP-93/05

March 1993

CRN-93-06

\footnotetext{
* Work supported in part by Deutsche Forschungsgemeinschaft and by Schweizerischer Nationalfonds.

$\dagger$ Heisenberg Fellow.
} 


\section{INTRODUCTION}

At low energies, chiral symmetry governs the interaction of the low-lying hadrons. To a good first approximation the current masses of the three light quarks can be set to zero and one can expand the QCD Green functions in powers of external momenta and quark masses around the so-called chiral limit. Assuming that the order parameter of chiral symmetry breaking, $B_{0}=-<0|\bar{u} u| 0>/ F_{\pi}^{2}$, is of order $1 \mathrm{GeV}$, an unambiguous scheme emerges in the meson sector [1]. In the baryon sector matters are more complicated. The low-lying baryons have a non-vanishing mass in the chiral limit, of the order of the chiral symmetry breaking scale $B_{0}$, which complicates the low-energy structure in the meson-baryon system considerable [2]. Making use of methods borrowed from heavy quark effective field theories, Jenkins and Manohar [3] proposed to consider the baryons as very heavy static sources (see also Gasser and Leutwyler [4] and Weinberg [5]). This allows to define velocity eigen-fields and to leading order the troublesome baryon mass term can be eliminated from the Dirac lagrangian. This procedure is completely equivalent to the well-known Foldy-Wouthuysen transformation used in QED to order operators by inverse powers of the mass of the Dirac field*. A further complication in the baryon sector is the closeness of the first resonance multiplet. While in the meson sector the vector mesons only appear above $770 \mathrm{MeV}$, the mass of the $\rho$-meson, the spin-3/2 decuplet is only separated by $230 \mathrm{MeV}$ (in average) from the spin- $1 / 2$ ground state octet. This raises the immediate question whether these spin- $3 / 2$ fields should be included in the effective field theory from the very beginning. Also ample phenomenological evidence from the nucleon sector exists which points towards the importance of the spin-3/2 fields. In ref.[7] the effective field theory was enlarged to include the decuplet. Furthermore, numerous calculations have been performed concerning baryon masses, hyperon non-leptonic decays [9], nucleon polarizabilities [10] and so on within this framework. All these calculations are done under the assumption that only the terms non-analytic in the quark masses are important and therefore carry a spurious dependence on the scale introduced in dimensional regularization. In addition, kaon and eta loop contributions can be large, as first pointed out by Bijnens, Sonoda and Wise [11]. However, it is argued that the decuplet contributions to a large extent cancel these large kaon and eta loop terms, leaving one with a fairly well behaved chiral expansion even in the three flavor case.

While one might content oneself with these rather positive results, a closer look at how they are obtained makes one feel uneasy about them. First, chiral perturbation theory is a systematic expansion meaning that to a given order one has to take into account all contributions, may they be from loops (and thus eventually non-analytic in the quark masses) or higher order contact terms. These are multiplied by a priori unknown coefficients, the so-called low-energy constants. Only the sum of loop and higher order contact terms is naturally scale independent. Second, many of the results based on the approach of taking only calculable loop diagrams with octet and decuplet

* For a systematic analysis in flavor $\mathrm{SU}(2)$, see ref.[6]. 
intermediate states only give consistent results if one uses the rather small $D$ and $F$ axial vector coupling proposed in ref.[7]. These values, to our opinion, are simply an artefact of the calculational procedure and do not reflect the results of a consistent chiral perturbation calculation.

To put the finger on the problem, we reconsider here the classical problem of the baryon masses and $\sigma$-terms, namely the $\pi N \sigma$-term and two $K N \sigma$-terms. This is essentially the scalar sector of baryon chiral perturbation theory. The first systematic analysis was performed by Gasser [12] and the long-standing problem with the $\pi N \sigma$ term has recently been resolved by Gasser, Leutwyler and Sainio [13]. They combined a dispersion-theoretical approach with chiral symmetry constraints to show that the $\sigma$-term shift $\sigma_{\pi N}\left(2 M_{\pi}^{2}\right)-\sigma_{\pi N}(0)$ is as large as $15 \mathrm{MeV}$. Therefore the empirical value $\sigma_{\pi N}\left(2 M_{\pi}^{2}\right) \simeq 60 \mathrm{MeV}$ determined from $\pi N$ scattering can be reconciled if the strange quark admixture in the proton is $y \simeq 0.2$, leading to a mass shift of $m_{s}<p|\bar{s} s| p>\simeq 130$ $\mathrm{MeV}$, considerably lower than expected from first order quark mass perturbation theory $[12,14]$ and anticipated in some models of dynamical chiral symmetry breaking [15]. Jenkins and Manohar have argued that the heavy fermion formulation with decuplet fields is indeed consistent with these values [16]. This, in fact, is the statement we wish to elaborate on. First we perform a complete calculation up to order $q^{3}$, which only involves intermediate octet states. At this order (one-loop approximation) one has three counterterms with a priori unknown but finite coefficients. These can be fixed from the octet masses $\left(m_{N}, m_{\Lambda}, m_{\Sigma}, m_{\Xi}\right)$ and the value $\sigma_{\pi N}(0)$ since one of the counter terms appears in the baryon mass formulae in such a way that it always can be lumped together with the average octet mass in the chiral limit. This allows us to predict the two $K N \sigma$-terms, $\sigma_{K N}^{(1)}(0)$ and $\sigma_{K N}^{(2)}(0)$ as well as the $\sigma$-term shifts to the respective Cheng-Dashen points and the matrix element $m_{s}<p|\bar{s} s| p>$. We then proceed and add the low-lying decuplet fields. Here we leave the consistent calculation since the first visible effect of the decuplet appears at order $q^{4}$. So, in principle we should account for a host of other terms. Our aim is, however, more modest. We simply want to check whether the assertions made in refs. $[7-10,16]$ can be considered sound. The first, and rather obvious, observation to be made is that the inclusion of the decuplet fields spoils the consistent chiral power counting. This can be traced back to the residual mass dependence which can not be removed from the lagrangian involving velocity-dependent fields. Stated differently, in the chiral limit the average decuplet and octet masses differ by a nonzero amount of a few hundred $\mathrm{MeV}$. This leads to complications similar to the ones in the relativistic formulation of baryon chiral perturbation theory (related to the finite value of the nucleon mass in the chiral limit) discussed in ref.[2]. Also, in the baryon mass spectrum one finds an infinite renormalization of the previously finite counter terms of chiral power two. These problems have not yet been spelled out in the literature. For the numerical results we refer the reader to later sections, where it will become clear that one has to work harder to get a consistent picture of heavy baryon CHPT beyond $q^{3}$. As a nice by-product, we find an $\mathrm{SU}(2)$ result which allows to explain the large shift $\sigma_{\pi N}\left(2 M_{\pi}^{2}\right)-\sigma_{\pi N}(0)$ in terms of the $\Delta(1232)$ states (modulo 
the many other unknown effects appearing at order $q^{4}$ or higher). It is important to differentiate between the pure SU(2) results (where the kaons and etas only contribute very little) and the SU(3) results, which are afflicted by potentially large cancellations between $K$ and $\eta$ loops on one side and decuplet contributions on the other side. It is conceivable that one should first address these genuine $\mathrm{SU}(2)$ results and understand the chiral expansion for them before one turns to the more complicated three flavor sector. A first step was done in ref.[6] and we will come back to this problem in a future publication. To close the introduction, let us point out that a review on baryon CHPT is available [17], another one on the heavy fermion formulation [18] and related aspects are discussed in the more recent CHPT review [19].

\section{FORMALISM}

\section{II.1. Baryon masses and $\sigma$-terms at next-to-leading order}

In this section, we will give the formalism necessary to discuss the baryon masses and $\sigma$-terms. Our starting point is the effective chiral lagrangian of the pseudoscalar Goldstone bosons coupled to octet baryons (we do not exhibit the standard meson lagrangian)

$$
\mathcal{L}_{\phi B}^{(1)}=\operatorname{Tr}(\bar{B} i v \cdot \mathcal{D} B)+D \operatorname{Tr}\left(\bar{B} S^{\mu}\left\{u_{\mu}, B\right\}\right)+F \operatorname{Tr}\left(\bar{B} S^{\mu}\left[u_{\mu}, B\right]\right)
$$

where the Goldstone fields $\phi$ are collected in the $S U(3)$ matrix

$$
U(x)=\exp \left[i \phi(x) / F_{p}\right], \quad u(x)=\sqrt{U(x)}, \quad u_{\mu}=i u^{\dagger} \nabla_{\mu} U u^{\dagger}
$$

and $F_{p}$ is the pseudoscalar decay constant in the chiral limit. $B$ is the standard $S U(3)$ matrix representation of the low-lying spin-1/2 baryons $\left(p, n, \Lambda, \Sigma^{0}, \Sigma^{ \pm}, \Xi^{0}, \Xi^{-}\right)$and $S_{\mu}$ is a covariant spin operator satisfying $v \cdot S=0$ and $S^{2}=(1-d) / 4$ in $d$ space-time dimensions. The two axial coupling constants $D$ and $F$ are subject to the constraint $D+F=g_{A}=1.26$. We work in the heavy mass formalism, which means that baryons are considered as static sources and equivalently their momenta decompose as

$$
p_{\mu}=m_{0} v_{\mu}+l_{\mu}
$$

with $m_{0}$ the average octet mass in the chiral limit, $v_{\mu}$ the baryon four-velocity $\left(v^{2}=1\right)$ and $l_{\mu}$ a small off-shell momentum. In this extreme non-relativistic limit one can define velocity dependent fields such that the troublesome baryon mass term disappears from the original Dirac lagrangian for the baryons. In the absence of the baryon mass term a consistent low-energy expansion can be derived. The baryon propagator reads $i /(v \cdot l+i \epsilon)$. The low energy expansion resulting from loops goes along with an expansion in inverse powers of the baryon mass $m_{0}$. As indicated by the superscript (1) in eq.(2.1), tree level diagrams calculated from the lowest order effective lagrangian are of order $q$, with $q$ denoting a genuine small momentum. At next-to-leading order one has to include the 
one-loop graphs using solely the vertices given by $\mathcal{L}_{\phi B}^{(1)}$ and additional chirally symmetric counterterms of order $q^{2}$ and $q^{3}$, since the one-loop graphs all have chiral power $q^{3}$. These contact terms are accompanied by a priori unknown coupling constants and have to be fixed phenomenologically. In the present context only counter terms of chiral power $q^{2}$ contribute which account for quark mass insertions,

$$
\mathcal{L}_{\phi B}^{(2)}=b_{D} \operatorname{Tr}\left(\bar{B}\left\{\chi_{+}, B\right\}\right)+b_{F} \operatorname{Tr}\left(\bar{B}\left[\chi_{+}, B\right]\right)+b_{0} \operatorname{Tr}(\bar{B} B) \operatorname{Tr}\left(\chi_{+}\right)
$$

with $\chi_{+}=u^{\dagger} \chi u^{\dagger}+u \chi^{\dagger} u$ and $\chi=2 B_{0}(\mathcal{M}+\mathcal{S})$ where $\mathcal{S}$ denotes the nonet of external scalar sources. As we will see later on, the constants $b_{D}, b_{F}$ and $b_{0}$ can be fixed from the knowledge of the baryon masses and the $\pi N \sigma$-term (or one of the $K N \sigma$-terms). The constant $b_{0}$ can not be determined from the baryon mass spectrum alone since it contributes to all octet members in the same way. To this order in the chiral expansion, any baryon mass takes the form

$m_{B}=m_{0}-\frac{1}{24 \pi F_{p}^{2}}\left[\alpha_{B}^{\pi} M_{\pi}^{3}+\alpha_{B}^{K} M_{K}^{3}+\alpha_{B}^{\eta} M_{\eta}^{3}\right]+\gamma_{B}^{D} b_{D}+\gamma_{B}^{F} b_{F}-2 b_{0}\left(M_{\pi}^{2}+2 M_{K}^{2}\right)$

The first term on the right hand side of eq.(2.5) is the average octet mass in the chiral limit, the second one comprises the Goldstone boson loop contributions and the third term stems from the counter terms eq.(2.4). Notice that the loop contribution is ultraviolet finite and non-analytic in the quark masses since $M_{\phi}^{3} \sim m_{q}^{3 / 2}$. The constants $b_{D}$, $b_{F}$ and $b_{s}$ are therefore finite. The numerical factors read

$$
\begin{aligned}
& \alpha_{N}^{\pi}=\frac{9}{4}(D+F)^{2}, \quad \alpha_{N}^{K}=\frac{1}{2}\left(5 D^{2}-6 D F+9 F^{2}\right), \quad \alpha_{N}^{\eta}=\frac{1}{4}(D-3 F)^{2} ; \\
& \alpha_{\Sigma}^{\pi}=D^{2}+6 F^{2}, \quad \alpha_{\Sigma}^{K}=3\left(D^{2}+F^{2}\right), \quad \alpha_{\Sigma}^{\eta}=D^{2} ; \\
& \alpha_{\Lambda}^{\pi}=3 D^{2}, \quad \alpha_{\Lambda}^{K}=D^{2}+9 F^{2}, \quad \alpha_{\Lambda}^{\eta}=D^{2} ; \\
& \alpha_{\Xi}^{\pi}=\frac{9}{4}(D-F)^{2}, \quad \alpha_{\Xi}^{K}=\frac{1}{2}\left(5 D^{2}+6 D F+9 F^{2}\right), \quad \alpha_{\Xi}^{\eta}=\frac{1}{4}(D+3 F)^{2} ; \\
& \gamma_{N}^{D}=-4 M_{K}^{2}, \quad \gamma_{N}^{F}=4 M_{K}^{2}-4 M_{\pi}^{2} ; \quad \gamma_{\Sigma}^{D}=-4 M_{\pi}^{2}, \quad \gamma_{\Sigma}^{F}=0 ; \\
& \gamma_{\Lambda}^{D}=-\frac{16}{3} M_{K}^{2}+\frac{4}{3} M_{\pi}^{2}, \quad \gamma_{\Lambda}^{F}=0 ; \quad \gamma_{\Xi}^{D}=-4 M_{K}^{2}, \quad \gamma_{\Xi}^{F}=-4 M_{K}^{2}+4 M_{\pi}^{2} .
\end{aligned}
$$

At this order, the deviation from the Gell-Mann-Okubo formula reads

$$
\begin{aligned}
\frac{1}{4}\left[3 m_{\Lambda}+m_{\Sigma}-2 m_{N}-2 m_{\Xi}\right] & =\frac{3 F^{2}-D^{2}}{96 \pi F_{p}^{2}}\left[M_{\pi}^{3}-4 M_{K}^{3}+3 M_{\eta}^{3}\right] \\
& =\frac{3 F^{2}-D^{2}}{96 \pi F_{p}^{2}}\left[M_{\pi}^{3}-4 M_{K}^{3}+\frac{1}{\sqrt{3}}\left(M_{K}^{2}-M_{\pi}^{2}\right)^{3 / 2}\right]
\end{aligned}
$$


where in the second line we have used the GMO relation for the $\eta$-meson mass, which is legitimate if one works at next-to-leading order.

Further information on the scalar sector is given by the scalar form factors or $\sigma$ terms which measure the strength of the various matrix-elements $m_{q} \bar{q} q$ in the proton. One defines:

$$
\begin{aligned}
\sigma_{\pi N}(t) & =\hat{m}<p^{\prime}|\bar{u} u+\bar{d} d| p> \\
\sigma_{K N}^{(1)}(t) & =\frac{1}{2}\left(\hat{m}+m_{s}\right)<p^{\prime}|\bar{u} u+\bar{s} s| p> \\
\sigma_{K N}^{(2)}(t) & =\frac{1}{2}\left(\hat{m}+m_{s}\right)<p^{\prime}|-\bar{u} u+2 \bar{d} d+\bar{s} s| p>
\end{aligned}
$$

with $t=\left(p^{\prime}-p\right)^{2}$ the invariant momentum transfer squared and $\hat{m}=\left(m_{u}+m_{d}\right) / 2$ the average light quark mass. At zero momentum transfer, the strange quark contribution to the nucleon mass is given by

$$
m_{s}<p|\bar{s} s| p>=\left(\frac{1}{2}-\frac{M_{\pi}^{2}}{4 M_{K}^{2}}\right)\left[3 \sigma_{K N}^{(1)}(0)+\sigma_{K N}^{(2)}(0)\right]+\left(\frac{1}{2}-\frac{M_{K}^{2}}{M_{\pi}^{2}}\right) \sigma_{\pi N}(0)
$$

making use of the leading order meson mass formulae $M_{\pi}^{2}=2 \hat{m} B_{0}$ and $M_{K}^{2}=(\hat{m}+$ $\left.m_{s}\right) B_{0}$ which are sufficiently accurate to the order we are working. The chiral expansion at next-to-leading order for the $\sigma$-terms reads

$$
\begin{aligned}
\sigma_{\pi N}(0)= & \frac{M_{\pi}^{2}}{64 \pi F_{p}^{2}}\left[-4 \alpha_{N}^{\pi} M_{\pi}-2 \alpha_{N}^{K} M_{K}-\frac{4}{3} \alpha_{N}^{\eta} M_{\eta}\right]-2 M_{\pi}^{2}\left(b_{D}+b_{F}+2 b_{0}\right) \\
\sigma_{K N}^{(j)}(0)= & \frac{M_{K}^{2}}{64 \pi F_{p}^{2}}\left[-2 \alpha_{N}^{\pi} M_{\pi}-3 \xi_{K}^{(j)} M_{K}-\frac{10}{3} \alpha_{N}^{\eta} M_{\eta}\right. \\
& \left.-2 \xi_{\pi \eta}^{(j)} \alpha_{N}^{\pi \eta} \frac{M_{\pi}^{2}+M_{\pi} M_{\eta}+M_{\eta}^{2}}{M_{\pi}+M_{\eta}}\right]+4 M_{K}^{2}\left(\xi_{D}^{(j)} b_{D}+\xi_{F}^{(j)} b_{F}-b_{0}\right)
\end{aligned}
$$

for $j=1,2$ with coefficients

$$
\begin{aligned}
& \xi_{K}^{(1)}=\frac{7}{3} D^{2}-2 D F+5 F^{2}, \quad \xi_{K}^{(2)}=3(D-F)^{2}, \quad \xi_{\pi \eta}^{(1)}=1, \quad \xi_{\pi \eta}^{(2)}=-3, \\
& \xi_{D}^{(1)}=-1, \quad \xi_{D}^{(2)}=0, \quad \xi_{F}^{(1)}=0, \quad \xi_{F}^{(2)}=1 ; \quad \alpha_{N}^{\pi \eta}=\frac{1}{3}(D+F)(3 F-D) .
\end{aligned}
$$

This completely determines the scalar sector at next-to-leading order. Note that the $\pi N$ $\sigma$-term is given as $\sigma_{\pi N}(0)=\hat{m}\left(\partial m_{N} / \partial \hat{m}\right)$ according to the Feynman-Hellman theorem. The shifts of the $\sigma$-terms from $t=0$ to the respective Cheng-Dashen points do not involve any contact terms,

$$
\begin{aligned}
\sigma_{\pi N}\left(2 M_{\pi}^{2}\right)-\sigma_{\pi N}(0)= & \frac{M_{\pi}^{2}}{64 \pi F_{p}^{2}}\left\{\frac{4}{3} \alpha_{N}^{\pi} M_{\pi}\right. \\
& +\frac{2}{3} \alpha_{N}^{K}\left[\frac{M_{\pi}^{2}-M_{K}^{2}}{\sqrt{2} M_{\pi}} \ln \frac{\sqrt{2} M_{K}+M_{\pi}}{\sqrt{2} M_{K}-M_{\pi}}+M_{K}\right] \\
& \left.+\frac{4}{9} \alpha_{N}^{\eta}\left[\frac{M_{\pi}^{2}-M_{\eta}^{2}}{\sqrt{2} M_{\pi}} \ln \frac{\sqrt{2} M_{\eta}-M_{\pi}}{\sqrt{2} M_{\eta}-M_{\pi}}+M_{\eta}\right]\right\}
\end{aligned}
$$




$$
\begin{aligned}
& \sigma_{K N}^{(j)}\left(2 M_{K}^{2}\right)-\sigma_{K N}^{(j)}(0)=\frac{M_{K}^{2}}{128 \pi F_{p}^{2}}\left\{\frac{4}{3} \alpha_{N}^{\pi}\left[\frac{M_{K}^{2}-M_{\pi}^{2}}{\sqrt{2} M_{K}}\left(\ln \frac{M_{K}+\sqrt{2} M_{\pi}}{M_{K}-\sqrt{2} M_{\pi}}+i \pi\right)+M_{\pi}\right]\right. \\
& +\frac{20}{9} \alpha_{N}^{\eta}\left[\frac{M_{K}^{2}-M_{\eta}^{2}}{\sqrt{2} M_{K}} \ln \frac{\sqrt{2} M_{\eta}+M_{K}}{\sqrt{2} M_{\eta}-M_{K}}+M_{\eta}\right]+2 \xi_{K}^{(j)} M_{K} \\
& \left.+\xi_{\pi \eta}^{(j)} \alpha_{N}^{\pi \eta}\left[\frac{2 M_{K}^{2}-M_{\pi}^{2}-M_{\eta}^{2}}{\sqrt{2} M_{K}} \ln \frac{\sqrt{2} M_{K}+M_{\pi}+M_{\eta}}{M_{\pi}+M_{\eta}-\sqrt{2} M_{K}}+2 \frac{M_{\pi}^{2}+M_{\eta}^{2}}{M_{\pi}+M_{\eta}}\right]\right\}
\end{aligned}
$$

Notice that the shifts of the two $K N \sigma$-terms acquire an imaginary part since the pion loop has a branch cut starting at $t=4 M_{\pi}^{2}$ which is below the kaon Cheng -Dashen point $t=2 M_{K}^{2}{ }^{*}$. In the limit of large kaon and eta mass the result eq.(2.11a) agrees, evidently, with the ancient calculation of Pagels and Pardee [20] once one accounts for the numerical error of a factor 2 in that paper. Clearly, the $\sigma$-term shifts are nonanalytic in the quark masses since they scale with the third power of the pseudoscalar meson masses. Our strategy will be the following: We use the empirically known baryon masses and the recently determined value of $\sigma_{\pi N}(0)$ [13] to fix the unknown parameters $m_{0}, b_{D}, b_{F}$ and $b_{0}$. This allows us to predict the two $K N \sigma$-terms $\sigma_{K N}^{(j)}(0)$. The shifts of the $\sigma$-terms are independent of this fit. Before presenting results, let us discuss the inclusion of the low-lying spin-3/2 decuplet in the effective theory.

\section{II.2. Inclusion of the decuplet-fields}

The low-lying decuplet is only separated by $\Delta=231 \mathrm{MeV}$ from the octet baryons, which is just $\frac{5}{3} M_{\pi}$ and considerably smaller than the kaon or eta mass. One therefore expects the excitations of these resonances to play an important role even at low energies. This is also backed by phenomenological models of the nucleon in which the $\Delta(1232)$ excitations play an important role. In the meson sector, the first resonances are the vector mesons $\rho$ and $\omega$ at about $800 \mathrm{MeV}$, i.e. they are considerably heavier than the Goldstone bosons. It was therefore argued by Jenkins and Manohar [7] to include the spin-3/2 decuplet in the effective theory from the start. Denote by $T^{\mu}$ a RaritaSchwinger fields in the heavy mass formulation satisfying $v \cdot T=0$. The effective lagrangian of the spin-3/2 fields at lowest order reads

$$
\mathcal{L}_{\phi B T}=-i \bar{T}^{\mu} v \cdot \mathcal{D} T_{\mu}+\Delta \bar{T}^{\mu} T_{\mu}+\frac{C}{2}\left(\bar{T}^{\mu} u_{\mu} B+\bar{B} u_{\mu} T^{\mu}\right) .
$$

where we have suppressed the flavor $S U(3)$ indices. Notice that there is a remaining mass dependence which comes from the average decuplet-octet splitting $\Delta$ which does not vanish in the chiral limit. The constant $C$ is fixed from the decay $\Delta \rightarrow N \pi$ or the

* Since we choose the GMO value for the $\eta$ mass, $M_{\pi}+M_{\eta}>\sqrt{2} M_{K}$, the $\pi \eta$ loop does not contribute to the imaginary part in eq.(2.11b). For the physical value of the $\eta$ mass this contribution is tiny compared to the pion loop. 
average of some strong decuplet decays. The decuplet propagator carries the information about the mass splitting $\Delta$ and reads

$$
\frac{i P_{\mu \nu}}{v \cdot l-\Delta+i \epsilon}
$$

with

$$
P_{\mu \nu}=v_{\mu} v_{\nu}-g_{\mu \nu}-4 \frac{d-3}{d-1} S_{\mu} S_{\nu}
$$

in $d$ dimensions. The projector obviously satisfies the constraints $v^{\mu} P_{\mu \nu}=P_{\mu \nu} v^{\nu}=0$ and $P_{\mu}^{\mu}=-2$. The appearance of the mass splitting $\Delta$ spoils the exact one-to one correspondence between the loop and low-energy expansion. Two scales $F_{p}$ and $\Delta$ which are both non-vanishing in the chiral limit enter the loop calculations and they can combine in the form $\left(\Delta / F_{p}\right)^{2}$. The breakdown of the consistent chiral counting in the presence of the decuplet is seen in the loop contribution to the baryon mass. The loop diagrams with intermediate decuplets states which naively count as order $q^{4}$ renormalize the average octet baryon mass even in the chiral limit by an infinite amount. Therefore one has to add a counter term of chiral power $q^{0}$ to keep the value $m_{0}$ fixed

$$
\begin{aligned}
\delta \mathcal{L}_{\phi B}^{(0)} & =-\delta m_{0} \operatorname{Tr}(\bar{B} B) \\
\delta m_{0} & =\frac{10}{3} \frac{C^{2} \Delta^{3}}{F_{p}^{2}}\left[L+\frac{1}{16 \pi^{2}}\left(\ln \frac{2 \Delta}{\lambda}-\frac{5}{6}\right)\right] \\
L & =\frac{\lambda^{d-4}}{16 \pi^{2}}\left[\frac{1}{d-4}+\frac{1}{2}\left(\gamma_{E}-\ln 4 \pi-1\right)\right]
\end{aligned}
$$

with $\lambda$ the scale introduced in dimensional regularization and $\gamma_{E}=0.577215 \ldots$ the EulerMascheroni constant. This mass shift is similar to the one in the relativistic version of pion-nucleon CHPT, where the non-vanishing nucleon mass in the chiral limit leads to similar complications.

Let us now turn to the baryon masses. The inclusion of the decuplet fields has two effects on the mass formulae eq.(2.5). First there is an infinite loop contribution with decuplet intermediate states and, second, an infinite renormalization of the order $q^{2}$ of the low-energy constants $b_{D}, b_{F}$ and $b_{0}$. Indeed this divergent mass shift due to decuplet loops has not been treated consistently before. To account for it, we give the following renormalization prescription for $b_{D}, b_{F}$ and $b_{0}$

$$
\begin{aligned}
& b_{D}=b_{D}^{r}(\lambda)-\frac{\Delta C^{2}}{2 F_{p}^{2}} L \\
& b_{F}=b_{F}^{r}(\lambda)+\frac{5 \Delta C^{2}}{12 F_{p}^{2}} L \\
& b_{0}=b_{0}^{r}(\lambda)+\frac{7 \Delta C^{2}}{6 F_{p}^{2}} L
\end{aligned}
$$


where the finite pieces $b_{D, F, 0}^{r}(\lambda)$ will be determined by our fitting procedure (see below). Therefore the decuplet contributions to the octet masses can be written in the form

$$
\delta m_{B}=\frac{C^{2}}{24 \pi^{2} F_{p}^{2}}\left[\beta_{B}^{\pi} H\left(M_{\pi}\right)+\beta_{B}^{K} H\left(M_{K}\right)+\beta_{B}^{\eta} H\left(M_{\eta}\right)\right]
$$

with coefficients

$$
\begin{aligned}
& \beta_{N}^{\pi}=4, \quad \beta_{N}^{K}=1, \quad \beta_{N}^{\eta}=0 ; \quad \beta_{\Sigma}^{\pi}=\frac{2}{3}, \quad \beta_{\Sigma}^{K}=\frac{10}{3}, \quad \beta_{\Sigma}^{\eta}=1 ; \\
& \beta_{\Lambda}^{\pi}=3, \quad \beta_{\Lambda}^{K}=2, \quad \beta_{\Lambda}^{\eta}=0 ; \quad \beta_{\Xi}^{\pi}=1, \quad \beta_{\Xi}^{K}=3, \quad \beta_{\Xi}^{\eta}=1 .
\end{aligned}
$$

and

$$
\begin{aligned}
& H\left(M_{\phi}\right)=\Delta^{3} \ln \frac{2 \Delta}{M_{\phi}}+\Delta M_{\phi}^{2}\left(\frac{3}{2} \ln \frac{M_{\phi}}{\lambda}-1\right)-\left(\Delta^{2}-M_{\phi}^{2}\right)^{3 / 2} \ln \left[\frac{\Delta}{M_{\phi}}+\sqrt{\left.\frac{\Delta^{2}}{M_{\phi}^{2}}-1\right]} ; M_{\phi}<\Delta\right. \\
& H\left(M_{\phi}\right)=\Delta^{3} \ln \frac{2 \Delta}{M_{\phi}}+\Delta M_{\phi}^{2}\left(\frac{3}{2} \ln \frac{M_{\phi}}{\lambda}-1\right)-\left(M_{\phi}^{2}-\Delta^{2}\right)^{3 / 2} \arccos \frac{\Delta}{M_{\phi}} ; \quad M_{\phi}>\Delta .
\end{aligned}
$$

It is instructive to expand $H\left(M_{\phi}\right)$ for small $M_{\phi}$

$$
H\left(M_{\phi}\right)=\frac{3}{4} \Delta M_{\phi}^{2}\left(2 \ln \frac{2 \Delta}{\lambda}-1\right)+\frac{3 M_{\phi}^{4}}{32 \Delta}\left(4 \ln \frac{M_{\phi}}{2 \Delta}-3\right)+\ldots
$$

This shows that the leading contribution of the diagrams with intermediate decuplet states is of order $M_{\phi}^{2}$, which means linear and therefore analytic in the quark masses. This again demonstrates the problems with the chiral power counting in the presence of a second non-vanishing scale $\Delta$. However, this contribution has no physical effect since it can be absorbed in the renormalized values of $b_{D, F, 0}^{r}(\lambda)$. So the first non-trivial effect of the decuplet states on the baryon masses appears at order $q^{4}$, which means beyond next-to-leading order. This is in agreement with the decoupling theorem [21]. Clearly, at this order there are many other contributions. We will come back to this point later on. The decuplet contribution to the GMO deviation reads

$$
\frac{1}{4}\left[3 \delta m_{\Lambda}+\delta m_{\Sigma}-2 \delta m_{N}-2 \delta m_{\Xi}\right]=\frac{C^{2}}{288 \pi^{2} F_{p}^{2}}\left[-H\left(M_{\pi}\right)+4 H\left(M_{K}\right)-3 H\left(M_{\eta}\right)\right]
$$

Notice that despite the appearence of the renormalization scale $\lambda$ in the various $H\left(M_{\phi}\right)$, the right hand side of eq.(2.18) is indeed scale independent due to the GMO relation for the $\eta$-mass. 
Similarly, the decuplet contributes to the $\sigma$-terms at $t=0$ are given by

$$
\begin{aligned}
\delta \sigma_{\pi N}(0) & =\frac{M_{\pi}^{2} C^{2}}{64 \pi^{2} F_{p}^{2}}\left[8 \tilde{H}\left(M_{\pi}\right)+\tilde{H}\left(M_{K}\right)\right] \\
\delta \sigma_{K N}^{(1)}(0) & =\frac{M_{K}^{2} C^{2}}{64 \pi^{2} F_{p}^{2}}\left[4 \tilde{H}\left(M_{\pi}\right)+\frac{4}{3} \tilde{H}\left(M_{K}\right)\right] \\
\delta \sigma_{K N}^{(2)}(0) & =\frac{M_{K}^{2} C^{2}}{64 \pi^{2} F_{p}^{2}}\left[4 \tilde{H}\left(M_{\pi}\right)+2 \tilde{H}\left(M_{K}\right)\right]
\end{aligned}
$$

with

$$
\begin{aligned}
& \tilde{H}\left(M_{\phi}\right)=\Delta\left(2 \ln \frac{M_{\phi}}{\lambda}-1\right)+2 \sqrt{\Delta^{2}-M_{\phi}^{2}} \ln \left[\frac{\Delta}{M_{\phi}}+\sqrt{\left.\frac{\Delta^{2}}{M_{\phi}^{2}}-1\right]} ; \quad M_{\phi}<\Delta\right. \\
& \tilde{H}\left(M_{\phi}\right)=\Delta\left(2 \ln \frac{M_{\phi}}{\lambda}-1\right)-2 \sqrt{M_{\phi}^{2}-\Delta^{2}} \arccos \frac{\Delta}{M_{\phi}} ; \quad M_{\phi}>\Delta .
\end{aligned}
$$

The contribution of the decuplet to the $\sigma$-term shifts can be most economically represented as a dispersion integral, the appropriate imaginary parts are collected in appendix A. However, for the later discussion, let us consider $\sigma_{\pi N}\left(2 M_{\pi}^{2}\right)-\sigma_{\pi N}(0)$ in $S U(2)$, i.e. retaining only $N$ and $\Delta(1232)$ intermediate states. One finds in this case

$$
\begin{array}{r}
\sigma_{\pi N}\left(2 M_{\pi}^{2}\right)-\sigma_{\pi N}(0)=\frac{3 g_{A}^{2} M_{\pi}^{4}}{16 \pi^{2} F_{\pi}^{2}} \int_{4 M_{\pi}^{2}}^{\infty} \frac{d t}{t^{3 / 2}\left(t-2 M_{\pi}^{2}\right)}\left[\frac{\pi}{4}\left(t-2 M_{\pi}^{2}\right)-\Delta \sqrt{t-4 M_{\pi}^{2}}\right. \\
\left.+\left(t-2 M_{\pi}^{2}+2 \Delta^{2}\right) \arctan \frac{\sqrt{t-4 M_{\pi}^{2}}}{2 \Delta}\right]
\end{array}
$$

where we used $C=\frac{3}{2} g_{A}$, coming from the $S U(4)$ relation between the $\pi N N$ and $\pi N \Delta$ coupling constants. This completes the necessary formalism. It is obvious from the discussion so far that the inclusion of the decuplet in baryon CHPT is an incomplete attempt since there are many other terms of order $q^{4}$ and higher. For example, Jenkins and Manohar $[8,16]$ have included tadpole diagrams with new vertices from $\mathcal{L}_{\phi B}^{(2)}$. These are of order $q^{4}$ and can give rise to non-analytic pieces like $m_{q}^{2} \ln m_{q}$. In what follows, we will not consider such diagrams but rather assume that anything at order $q^{4}$ is modelled by the inclusion of the low-lying spin-3/2 baryons. This can, of course, not substitute a full scale $q^{4}$ calculation including all terms at this order, but allows us to critically examine the role of the decuplet fields since their contribution is unique. We are motivated by the many papers making use of the Jenkins-Manohar proposal and want to see to what extent such an approximation is a good thing to do. 


\section{RESULTS}

In this section, we will first present results for the complete $q^{3}$ calculation outlined in section II and then proceed to add the decuplet.

\section{III.1. Results at order $\mathcal{O}\left(q^{3}\right)$}

First, we must fix parameters. Throughout, we us $M_{\pi}=138 \mathrm{MeV}, M_{K}=495$ $\mathrm{MeV}$ and $M_{\eta}^{2}=\left(4 M_{K}^{2}-M_{\pi}^{2}\right) / 3=(566 \mathrm{MeV})^{2}$ as given by the GMO relation for the pseudoscalar mesons. This is a consistent procedure since the differences to the physical $\eta$ mass only shows up at higher order. For the pseudoscalar decay constant, we can either use $F_{\pi}=92.6 \mathrm{MeV}$ or $F_{K}=112 \mathrm{MeV}$. Mostly, we use an average value $F_{p}=\left(F_{\pi}+F_{K}\right) / 2 \simeq 100 \mathrm{MeV}$. Since in all terms $F_{p}^{2}$ appears, we will vary $F_{p}$ from $F_{\pi}$ to $F_{K}$ to find out how sensitive the results are to this higher order effect (the difference of $F_{\pi}$ and $F_{K}$ in the meson sector is of order $q^{4}$ ). Furthermore, we use $F=0.5$ and $D=0.75$, which leads to $g_{A}=1.25$. Two other sets of $D$ and $F$ values, the one of Bourquin et al. [22], $F=0.477$ and $D=0.756$, and the central value of Jaffe and Manohar [23], $F=0.47$ and $D=0.81$. The four unknowns, which are the three lowenergy constants $b_{D}, b_{F}$ and $b_{0}$ and the average octet mass (in the chiral limit) $m_{0}$ are obtained from a least square fit to the physical baryon masses $(N, \Sigma, \Lambda, \Xi)$ and the value of $\sigma_{\pi N}(0) \simeq 45 \mathrm{MeV}$. This allows to predict $\sigma_{K N}^{(1)}(0)$ and $\sigma_{K N}^{(2)}(0)$ and the much discussed matrix element $m_{s}<p|\bar{s} s| p>$, i.e. the contribution of the strange quarks to the nucleon mass. We also give the value of the GMO deviation $\left(3 m_{\Lambda}+m_{\Sigma}-2 m_{N}-2 m_{\Xi}\right) / 4$, which experimentally is $6.5 \mathrm{MeV}$.

The results of this complete $\mathcal{O}\left(q^{3}\right)$ calculation are shown in table 1 . The dependence on the values of the $D$ and $F$ axial vector constants is rather weak, only in the case of the central values of ref.[23] an accidental cancellation occurs $\left(D^{2} \simeq 3 F^{2}\right)$ which makes the GMO deviation very small. In most of the other cases one gets roughly half of the empirical value. However, notice that it is a very small number on the typical baryon mass scale of $1 \mathrm{GeV}$ and can therefore not expected to be predicted accurately. The strangeness matrix element in most cases is negative and of reasonable magnitude of about $200 \mathrm{MeV}$. Within the accuracy of the calculation, the $K N \sigma$-terms turn out to be

$$
\begin{aligned}
\sigma_{K N}^{(1)}(0) & \simeq 200 \pm 50 \mathrm{MeV} \\
\sigma_{K N}^{(2)}(0) & \simeq 140 \pm 40 \mathrm{MeV}
\end{aligned}
$$

which is comparable to the first order perturbation theory analysis having no strange quarks, $\sigma_{K N}^{(1)}(0)=205 \mathrm{MeV}$ and $\sigma_{K N}^{(2)}(0)=63 \mathrm{MeV}$ [24]. Clearly, if one varies the value of $\sigma_{\pi N}(0)$ by $\pm 10 \mathrm{MeV}$, the results are rather different. This shows up in a value of $b_{0}$ which changes from -0.62 to $-0.88 \mathrm{GeV}^{-1}$ which has quite a dramatic impact on the $K N \sigma$-terms and the value of $m_{s}<p|\bar{s} s| p>$. For our analysis, however, we take the central value of $\sigma_{\pi N}(0)=45 \mathrm{MeV}$ [13] as given. Clearly a more accurate determination of this fundamental quantity would be very much needed. We also have performed a calculation with $M_{\eta}=549 \mathrm{MeV}$, the results are very close to the ones for $M_{\eta}$ given by 
the GMO relation (for the same $D, F$ and $F_{p}$ ) with the exception of the GMO deviation for the baryon masses.

The $\sigma$-term shifts are given by $\sigma_{\pi N}\left(2 M_{\pi}^{2}\right)-\sigma_{\pi N}(0)=7.4 \mathrm{MeV}[6,20]$, which is half of the empirical value found in ref.[13]. We will come back to this point later on. Furthermore, one finds

$$
\begin{aligned}
& \sigma_{K N}^{(1)}\left(2 M_{K}^{2}\right)-\sigma_{K N}^{(1)}(0)=(271+i 303) \mathrm{MeV} \\
& \sigma_{K N}^{(2)}\left(2 M_{K}^{2}\right)-\sigma_{K N}^{(2)}(0)=(21+i 303) \mathrm{MeV}
\end{aligned}
$$

whose real part can be estimated simply $\operatorname{Re}\left(\sigma_{K N}^{(1)}\left(2 M_{K}^{2}\right)-\sigma_{K N}^{(1)}(0)\right) \simeq\left[\sigma_{\pi N}\left(2 M_{\pi}^{2}\right)-\right.$ $\left.\sigma_{\pi N}(0)\right]\left(M_{K} / M_{\pi}\right)^{3}=7.4 \cdot 42.2 \mathrm{MeV}=340 \mathrm{MeV}$. The rather small real part in $\Delta \sigma_{K N}^{(2)}$ stems from the large negative contribution of the $\pi \eta$-loop which leads to strong cancellations. Notice the large imaginary parts in $\sigma_{K N}^{(j)}\left(2 M_{K}^{2}\right)-\sigma_{K N}^{(j)}(0)$ due to the two-pion cut.

\section{III.2. Results with inclusion of the decuplet}

In the case of adding the decuplet, we will first keep the value of the pseudoscalar decay constant $F_{p}=100 \mathrm{MeV}$ fixed. For the mass splitting we use either $\Delta=231 \mathrm{MeV}$ or $\Delta=293 \mathrm{MeV}$ (from the $N \Delta$ splitting) and the value of $C$ is given to be 1.8 from the strong decay $\Delta \rightarrow N \pi$ and $C=1.5$ from an overall fit to the decuplet decays [18]. The scale of dimensional regularization is chosen at $\lambda=1 \mathrm{GeV}$ [6]. It plays of course no role in the physical results, but it should be kept in mind that the scale dependent values of $b_{D, F, 0}(\lambda)$ are given at this scale. For the values of $D$ and $F$, we will use our central ones $(F=0.5, D=0.75)$ and also show results with the small values of ref.[7], $D=0.56, F=2 D / 3$ together with $C=2 D=1.12[16]$.

In table 2, we show the results for the full decuplet contribution according to eqs.(2.16) and (2.19) for the baryon masses and $\sigma$-terms, respectively. For comparison, table 3 gives the results accounting only for the leading term at order $q^{4}$ arising from the decuplet intermediate states, making use of eq.(2.17) and the expanded form of eq.(2.19). First, one notices that for physical values of the $F$ and $D$ constants, an inconsistent picture emerges. While the analysis of the $\pi N \sigma$-term leads one to believe that the strange quark contributes of the order of $15 \%$ to the proton mass, this is completely different when the decuplet is included. Comparison of table 2 and 3 shows that the bulk of this effect comes from the terms of order $q^{4}$. The conclusion drawn from this exercise is that simply taking the decuplet fields at order $q^{4}$ is meaningless. It still might be possible as argued in ref.[16] that despite a large contribution at order $M_{K}^{4} \sim q^{4}$, the chiral series might converge with the small parameter $\left(M_{K} / 4 \pi F_{\pi}\right)^{2}=0.16$. However, it is clear that only a complete calculation at order $q^{4}$ (and beyond) can give a definite answer to this question. Using the small values of $F, D$ and $C[7,16]$, we essentially recover the results of Jenkins and Manohar. The differences stem from the fact that we did not account for the tadpole diagrams with one insertion of $\mathcal{L}_{\phi B}^{(2)}$, eq.(2.4). In 
the spirit of the previous remarks, this is consistent. It is interesting to note that the results for $\sigma_{K N}^{(j)}(0)$ are quite similar to the ones of the full $q^{3}$ analysis if one uses the small values of $F, D$ and $C$.

Let us now consider the $\pi N \sigma$-term shift $\sigma_{\pi N}\left(2 M_{\pi}^{2}\right)-\sigma_{\pi N}(0)$. For the preferred choice $F=0.5, D=0.76$ and $C=\frac{3}{2} g_{A}$ based on the coupling constant relation $g_{\pi N \Delta}=$ $3 g_{\pi N} / \sqrt{2}$, we find

$$
\sigma_{\pi N}\left(2 M_{\pi}^{2}\right)-\sigma_{\pi N}(0)=15 \mathrm{MeV}
$$

which agrees nicely with the empirical result of ref.[13]. It is interesting to discuss this result. While the leading non-analytic piece proportional to $M_{\pi}^{3}$ gives $7.4 \mathrm{MeV}$, the same amount comes from the the analogous diagram with a $\Delta$ intermediate state. The kaon and $\eta$ loops add a meager $1.1 \mathrm{MeV}$, i.e. they are essentially negligible. This result agrees with the phenomenological analysis of Jameson et al.. We should stress that the spectral distribution $\operatorname{Im} \sigma_{\pi N}(t) / t^{2}$ is much less pronounced around $\sqrt{t}=600$ $\mathrm{MeV}$ than in ref.[13] but has a longer tail, so the total result remains the same. The $\Delta$-contribution mocks up the higher loop corrections of the dispersive analysis of Gasser et al. [13]. A similar phenomenon is also observed in the calculation of the nuclear forces to order $q^{4}$ in heavy baryon CHPT [26]. There, the intermediate range attraction comes from the uncorrelated two-pion exchange and some four-nucleon contact terms, whereas the phenomenological wisdom is that correlated two-pion exchange (also with diagrams involving intermediate $\Delta$ 's) is at the origin of this effect. For a more detailed discussion, see ref.[27]. Clearly, the result eq.(3.3) should be considered a curious accident since the correction to the leading term and the latter are of the same magnitude. It remains to be seen how other $q^{4}$ effects and higher order corrections not yet accounted for will modify eq.(3.3). It is, however, important to note the essential difference to the calculation of the baryon masses and of $\sigma_{K N}^{(j)}(0)$. In $\sigma_{\pi N}\left(2 M_{\pi}^{2}\right)-\sigma_{\pi N}(0)$, the heavy meson loops are irrelevant, i.e. it is an $\mathrm{SU}(2)$ statement. It therefore has a better chance to survive higher order loop corrections since the expansion parameter is $\left(M_{\pi} / 4 \pi F_{\pi}\right)^{2}=0.014$. Of course, there are also extra contact terms which will have to be evaluated. Finally, let us notice that the $K N \sigma$-terms shifts are large and that for the small values of $F, D$ and $C[16] \sigma_{\pi N}\left(2 M_{\pi}^{2}\right)-\sigma_{\pi N}(0)$ is only $6.8 \mathrm{MeV}$.

\section{SUMMARY AND OUTLOOK}

We have investigated the scalar sector of three-flavor baryon chiral perturbation theory. The baryons were treated as very massive fields, which allows to eliminate the troublesome mass term from the lowest order effective meson-baryon Lagrangian. Our findings can be summarized as follows:

- At next-to-leading order, i.e. order $\mathcal{O}\left(q^{3}\right)$ in the chiral expansion, one has three finite counterterms which amount to quark mass insertions. The respective lowenergy constants are denoted $b_{D}, b_{F}$ and $b_{0}$. Their values can be determined from a least square-fit to the baryon masses and the pion-nucleon $\sigma$-term at $t=0$. 
This allows to predict the two kaon-nucleon $\sigma$-terms, $\sigma_{\mathrm{KN}}^{(1,2)}(0)$. The values given in eq.(3.1) are not very different from the lowest order analysis. The shifts to the kaon Cheng-Dashen point are complex with a large real and large imaginary part, the latter being due to the two-pion cut. These numbers are considerably larger than the ones estimated by Gensini [28] a decade ago.* At this order, there is a one-to-one correspondence between the meson loop and small momentum expansion.

- We have then proceeded and added the low-lying spin-3/2 decuplet to the effective theory. We show that the new mass scale, which is the average octet-decuplet splitting, is non-vanishing in the chiral limit and thus induces an infinite renormalization of the baryon self-energies. This is analogous to the infinite mass shift in the relativistic formulation of baryon CHPT as spelled out by Gasser et al.[2]. The consistent power counting scheme is therefore not present any longer. Similarly, there is also an infinite renormalization of the three low-energy constant from the $\mathcal{O}\left(q^{2}\right)$ effective meson-baryon Lagrangian. Dissecting the contributions from the diagrams with intermediate decuplet states, one finds to leading order self-energy contributions which are proportional to the quark masses. However, these can be absorded entirely in the finite values of the renormalized low-energy constants $b_{D, F, 0}^{r}$. The first non-trivial effect of the decuplet states on the baryon masses appears at order $\mathcal{O}\left(q^{4}\right)$.

- The numerical evaluation of the decuplet contributions to the baryon masses and $\sigma$ terms shows a strong dependence on the values of $F, D$ and $C$, the latter one being related to the strong decuplet-octet-meson couplings. The decuplet contributions are large and for the physical values of $F$ and $D$, one does not have a consistent picture of the scalar sector of baryon CHPT. We disagree with the conclusion of refs. $[8,16,18]$ that small values of $F$ and $D$ lead to a consistent picture at this order. First, these values stem from an incomplete calculation and, second, at order $\mathcal{O}\left(q^{4}\right)$ there are many other diagrams which we (and other authors) did not take into account. The diagrams with intermediate decuplet states contribute to all orders in $q^{2}$, but dominantly at $\mathcal{O}\left(q^{4}\right)$ as comparison of tables 2 and 3 reveals. We conclude that it is not sufficient to include the decuplet to get an accurate machinery for baryon CHPT in the three flavor sector.

- As an interesting by-product, we have found that intermediate $\Delta(1232)$ states give a contribution to the $\pi N \sigma$-term shift as large as the leading order result of $7.5 \mathrm{MeV}$, so in total one has $15 \mathrm{MeV}$ in agreement with the result of ref.[13]. This is an $S U(2)$ number not affected by large kaon and eta loop contributions. However, at order $\mathcal{O}\left(q^{4}\right)$ there are other contributions not considered here which might invalidate this result. From this we conclude that it is mandatory to first understand in better

* For an update on the various extractions of the $\mathrm{KN} \sigma$-terms and a discussion of these results see ref.[29]. 
detail the two-flavor sector of baryon CHPT before one can hope to have a wellcontrolled chiral expansion including also the strange quark.

\section{APPENDIX: IMAGINARY PARTS OF SCALAR FORM FACTORS}

Here, we give explicit formulae for the imaginary parts to one loop of the three proton scalar form factors defined in eq.(2.8):

$$
\begin{aligned}
\operatorname{Im} \sigma_{\pi N}(t)= & \frac{M_{\pi}^{2}}{128 F_{p}^{2} \sqrt{t}}\left\{3(D+F)^{2}\left(t-2 M_{\pi}^{2}\right) \theta\left(t-4 M_{\pi}^{2}\right)\right. \\
& +\left(\frac{5}{3} D^{2}-2 D F+3 F^{2}\right)\left(t-2 M_{K}^{2}\right) \theta\left(t-4 M_{K}^{2}\right)+\left(\frac{D}{3}-F\right)^{2}\left(t-2 M_{\eta}^{2}\right) \theta\left(t-4 M_{\eta}^{2}\right) \\
& +\frac{16 C^{2}}{3 \pi}\left[-\Delta \sqrt{t-4 M_{\pi}^{2}}+\left(t-2 M_{\pi}^{2}+2 \Delta^{2}\right) \arctan \frac{\sqrt{t-4 M_{\pi}^{2}}}{2 \Delta}\right] \theta\left(t-4 M_{\pi}^{2}\right) \\
& \left.+\frac{2 C^{2}}{3 \pi}\left[-\Delta \sqrt{t-4 M_{K}^{2}}+\left(t-2 M_{K}^{2}+2 \Delta^{2}\right) \arctan \frac{\sqrt{t-4 M_{K}^{2}}}{2 \Delta}\right] \theta\left(t-4 M_{K}^{2}\right)\right\}
\end{aligned}
$$

$$
\begin{aligned}
& \operatorname{Im}_{N}^{(j)}(t)=\frac{M_{K}^{2}}{128 F_{p}^{2} \sqrt{t}}\left\{3(D+F)^{2}\left(\frac{t}{2}-M_{\pi}^{2}\right) \theta\left(t-4 M_{\pi}^{2}\right)+5\left(\frac{D}{3}-F\right)^{2}\left(\frac{t}{2}-M_{\eta}^{2}\right) \theta\left(t-4 M_{\eta}^{2}\right)\right. \\
& +\xi_{\pi \eta}^{(j)}(D+F)\left(F-\frac{D}{3}\right)\left(t-M_{\pi}^{2}-M_{\eta}^{2}\right) \theta\left(t-\left(M_{\pi}+M_{\eta}\right)^{2}\right)+\xi_{K}^{(j)}\left(t-2 M_{K}^{2}\right) \theta\left(t-4 M_{K}^{2}\right) \\
& \quad+\frac{8 C^{2}}{3 \pi}\left[-\Delta \sqrt{t-4 M_{\pi}^{2}}+\left(t-2 M_{\pi}^{2}+2 \Delta^{2}\right) \arctan \frac{\sqrt{t-4 M_{\pi}^{2}}}{2 \Delta}\right] \theta\left(t-4 M_{\pi}^{2}\right) \\
& \left.\quad+\left(2+\delta_{2 j}\right) \frac{4 C^{2}}{9 \pi}\left[-\Delta \sqrt{t-4 M_{K}^{2}}+\left(t-2 M_{K}^{2}+2 \Delta^{2}\right) \arctan \frac{\sqrt{t-4 M_{K}^{2}}}{2 \Delta}\right] \theta\left(t-4 M_{K}^{2}\right)\right\}
\end{aligned}
$$

The shifts of the $\sigma$-terms from $t=0$ to the respective Cheng-Dashen points are most economically represented in the form of a once-subtracted dispersion relation.

$$
\begin{aligned}
\sigma_{\pi N}\left(2 M_{\pi}^{2}\right)-\sigma_{\pi N}(0) & =\frac{2 M_{\pi}^{2}}{\pi} \int_{4 M_{\pi}^{2}}^{\infty} d t \frac{\operatorname{Im} \sigma_{\pi N}(t)}{t\left(t-2 M_{\pi}^{2}\right)} \\
\operatorname{Re}\left[\sigma_{K N}^{(j)}\left(2 M_{K}^{2}\right)-\sigma_{K N}^{(j)}(0)\right] & =\frac{2 M_{K}^{2}}{\pi} \mathcal{P} \int_{4 M_{\pi}^{2}}^{\infty} d t \frac{\operatorname{Im} \sigma_{K N}^{(j)}(t)}{t\left(t-2 M_{K}^{2}\right)}
\end{aligned}
$$

\section{REFERENCES}

1. J. Gasser and H. Leutwyler, Ann. Phys. (N.Y.) 158 (1984) 142; Nucl. Phys. B250 (1985) 465 . 
2. J. Gasser, M.E. Sainio and A. Švarc, Nucl. Phys. B307 (1988) 779.

3. E. Jenkins and A.V. Manohar, Phys. Lett. B255 (1991) 558.

4. J. Gasser and H. Leutwyler, Phys. Reports C87 (1982) 77.

5. S. Weinberg, Nucl. Phys. B363 (1991) 3.

6. V. Bernard, N. Kaiser, J. Kambor and Ulf-G. Meißner, Nucl. Phys. B388 (1992) 315 .

7. E. Jenkins and A.V. Manohar, Phys. Lett. B259 (1991) 353.

8. E. Jenkins, Nucl. Phys. B368 (1992) 190.

9. E. Jenkins, Nucl. Phys. B375 (1992) 561.

10. M.N. Butler and M.J. Savage, Phys. Lett. B294 (1992) 369.

11. J. Bijnens, H. Sonoda and M.B. Wise, Nucl. Phys. B261 (1985) 185.

12. J. Gasser, Ann. Phys. (N.Y.) 136 (1981) 62.

13. J. Gasser, H. Leutwyler and M.E. Sainio, Phys. Lett. 253B (1991) 252, 260.

14. J.F. Donoghue and C.R. Nappi, Phys. Lett. B168 (1986) 105.

15. V. Bernard, R.L. Jaffe and Ulf-G. Meißner, Nucl. Phys. B308 (1988) 753.

16. E. Jenkins and A.V. Manohar, Phys. Lett. B281 (1992) 336.

17. Ulf-G. Meißner, Int. J. Mod. Phys. E1 (1992) 561.

18. E. Jenkins and A.V. Manohar, in "Effective field theories of the standard model", ed. Ulf-G. Meißner, World Scientific, Singapore, 1992.

19. Ulf-G. Meißner, "Recent Developments in Chiral Perturbation Theory", Bern University preprint BUTP-93/01, 1993.

20. H. Pagels and W. Pardee, Phys. Rev. D4 (1971) 3225.

21. J. Gasser and A. Zepeda, Nucl. Phys. B174 (1980) 445.

22. M. Bourquin et al., Z. Phys. C21 (1983) 27.

23. R.L. Jaffe and A.V. Manohar, Nucl. Phys. B337 (1990) 509.

24. R.L. Jaffe and C. Korpa, Comm. Nucl. Part. Phys. 17 (1987) 163.

25. I. Jameson, A.W. Thomas and G. Chanfray, J. Phys. G: Nucl. Part. Phys. 18 (1992) L159.

26. C. Ordonez and U. van Kolck, Phys. Lett. B291 (1992) 459.

27. Ulf-G. Meißner, Comm. Nucl. Part. Phys. 20 (1991) 119.

28. P.M. Gensini, J. Phys. G: Nucl. Part. Phys. 7 (1981) 1177.

29. P.M. Gensini, in $\pi N$ Newsletter no. 6, eds. R.E. Cutkowsky, G. Höhler, W. Kluge and B.M.K. Nefkens, April 1992. 


\begin{tabular}{|ccc|ccccccccc|}
\hline$D$ & $F$ & $\begin{array}{c}F_{p} \\
{[\mathrm{MeV}]}\end{array}$ & $b_{D}$ & $b_{F}$ & $b_{0}$ & $m_{0}$ & $\sigma_{K N}^{(1)}(0)$ & $\sigma_{K N}^{(2)}(0)$ & SME & GMO \\
& {$\left[\mathrm{GeV}^{-1}\right]$} & {$\left[\mathrm{GeV}^{-1}\right]$} & {$\left[\mathrm{GeV}^{-1}\right]$} & {$[\mathrm{GeV}]$} & {$[\mathrm{MeV}]$} & {$[\mathrm{MeV}]$} & {$[\mathrm{MeV}]$} & {$[\mathrm{MeV}]$} \\
\hline 0.75 & 0.50 & 100 & 0.016 & -0.553 & -0.750 & 0.965 & 195.3 & 143.9 & -206 & 3.8 \\
0.756 & 0.477 & 100 & 0.037 & -0.540 & -0.753 & 0.958 & 204.3 & 146.0 & -192 & 2.3 \\
0.81 & 0.47 & 100 & 0.065 & -0.558 & -0.789 & 0.981 & 189.0 & 129.8 & -222 & 0.1 \\
0.75 & 0.50 & 92.6 & 0.008 & -0.610 & -0.788 & 1.014 & 154.0 & 117.4 & -278 & 4.5 \\
0.75 & 0.50 & 112 & 0.027 & -0.483 & -0.703 & 0.904 & 245.8 & 176.4 & -117 & 3.0 \\
\hline
\end{tabular}

Table 1: Results of the complete $\mathcal{O}\left(q^{3}\right)$ calculation. The values of $D, F$ and $F_{p}$ are input. GMO denotes the combination $\left(3 m_{\Lambda}+m_{\Sigma}-2 m_{N}-2 m_{\Xi}\right) / 4$ of the octet baryon masses. SME stands for the matrix element $m_{s}<p|\bar{s} s| p>$.

\begin{tabular}{|cccc|ccccccccc|}
\hline$D$ & $F$ & $\Delta$ & $C$ & $b_{D}$ & $b_{F}$ & $b_{0}$ & $m_{0}$ & $\sigma_{K N}^{(1)}(0)$ & $\sigma_{K N}^{(2)}(0)$ & SME & GMO \\
& & {$[\mathrm{MeV}]$} & & {$\left[\mathrm{GeV}^{-1}\right]$} & {$\left[\mathrm{GeV}^{-1}\right]$} & {$\left[\mathrm{GeV}^{-1}\right]$} & {$[\mathrm{GeV}]$} & {$[\mathrm{MeV}]$} & {$[\mathrm{MeV}]$} & {$[\mathrm{MeV}]$} & {$[\mathrm{MeV}]$} \\
\hline 0.75 & 0.50 & 293 & 1.8 & 0.623 & -1.057 & -1.744 & 1.330 & -39.4 & -111.5 & -667 & 11.5 \\
0.75 & 0.50 & 293 & 1.5 & 0.438 & -0.903 & -1.441 & 1.218 & 32.3 & -33.4 & -526 & 9.2 \\
0.75 & 0.50 & 231 & 1.8 & 0.642 & -1.072 & -1.752 & 1.360 & -58.2 & -131.6 & -704 & 12.0 \\
0.75 & 0.50 & 231 & 1.5 & 0.451 & -0.914 & -1.446 & 1.239 & 19.3 & -47.4 & -551 & 9.5 \\
0.56 & $2 D / 3$ & 293 & $2 D$ & 0.273 & -0.596 & -1.033 & 0.975 & 214.3 & 115.6 & -192 & 5.1 \\
0.56 & $2 D / 3$ & 231 & $2 D$ & 0.281 & -0.602 & -1.036 & 0.986 & 207.0 & 107.8 & -206 & 5.3 \\
\hline
\end{tabular}

Table 2: Results of the calculation including the full decuplet intermediate states. The values of $D, F, \Delta$ and $C$ are input. 


\begin{tabular}{|cccc|ccccccccc|}
\hline$D$ & $F$ & $\Delta$ & $C$ & $b_{D}$ & $b_{F}$ & $b_{0}$ & $m_{0}$ & $\sigma_{K N}^{(1)}(0)$ & $\sigma_{K N}^{(2)}(0)$ & SME & GMO \\
& & {$[\mathrm{MeV}]$} & & {$\left[\mathrm{GeV}^{-1}\right]$} & {$\left[\mathrm{GeV}^{-1}\right]$} & {$\left[\mathrm{GeV}^{-1}\right]$} & {$[\mathrm{GeV}]$} & {$[\mathrm{MeV}]$} & {$[\mathrm{MeV}]$} & {$[\mathrm{MeV}]$} & {$[\mathrm{MeV}]$} \\
\hline 0.75 & 0.50 & 293 & 1.8 & 0.547 & -0.995 & -1.696 & 1.222 & 38.2 & -23.3 & -513 & 7.0 \\
0.75 & 0.50 & 293 & 1.5 & 0.385 & -0.860 & -1.407 & 1.143 & 86.2 & 27.8 & -419 & 6.0 \\
0.75 & 0.50 & 231 & 1.8 & 0.518 & -0.971 & -1.673 & 1.185 & 67.0 & 10.3 & -455 & 4.8 \\
0.75 & 0.50 & 231 & 1.5 & 0.365 & -0.843 & -1.391 & 1.117 & 106.1 & 51.1 & -379 & 4.5 \\
0.56 & $2 D / 3$ & 293 & $2 D$ & 0.244 & -0.572 & -1.014 & 0.933 & 244.3 & 149.7 & -132 & 3.4 \\
0.56 & $2 D / 3$ & 231 & $2 D$ & 0.233 & -0.563 & -1.005 & 0.919 & 255.4 & 162.8 & -110 & 2.5 \\
\hline
\end{tabular}

Table 3: Same as in table 2 but with the decuplet contributions expanded up to and including $\mathcal{O}\left(q^{4}\right)$. 